\title{
Evidence for Presynaptic Inhibition of the Olfactory Commissural Pathway by Cholinergic Agonists and Stimulation of the Nucleus of the Diagonal Band
}

\author{
W. T. Nickell and M. T. Shipley \\ Department of Anatomy and Cell Biology, University of Cincinnati College of Medicine, Cincinnati, Ohio 45267-0521
}

We have investigated the role of the projection from the magnocellular basal forebrain to the olfactory bulb in regulating synaptic transmission in the commissural connection between the two olfactory bulbs.

Commissural fibers arise in the contralateral anterior olfactory nucleus, travel in the anterior wing of the anterior commissure (AC), and terminate in the granule cell layer of the olfactory bulb. Electrical stimulation of the commissure causes synaptic activation of granule cells in the granule cell layer of the bulb; the resulting field potential is a reliable indicator of this synaptic current. Microinjections of cholinergic agonists, but not of identical, or larger, quantities of vehicle, reduced the amplitude of this $A C$ field potential. Systemic injection of scopolamine reversed this depression and returned the $A C$ response amplitude to control levels. Irreversible AChE inhibition also reduced the amplitude of the AC response, and muscarinic blockade reversed this effect.

Cholinergic terminals in the olfactory bulb arise entirely from the axons of magnocellular basal forebrain neurons in the nucleus of the diagonal band (NDB). Electrical stimulation of NDB, which should release $A C h$, as well as other transmitters, depressed the AC response. Brief trains of NDB shocks caused a moderate decrease in the $A C$ response that lasted 1-2 sec. Longer shock trains, which caused marked potentiation of the NDB field potential, caused a profound, prolonged ( $>20 \mathrm{sec}$ ) inhibition of the $A C$ response. Antidromic tests demonstrated that NDB stimulation significantly decreased the excitability of AC terminals. This and other characteristics of the inhibition strongly suggest that the decrease in amplitude of the field potential response to AC stimulation caused by cholinergic agonists and stimulation of NDB is due to presynaptic inhibition leading to reduced release of transmitter from AC terminals.

These results suggest that one function of the basal forebrain projection to the olfactory bulb is inhibition of the commissural connection between the two olfactory bulbs. As NDB has been implicated in theta pacemaker input to the olfactory bulb, phasic NDB inhibition of centrifugal afferents to the bulb could function to coordinate signal processing

Received May 5, 1992; revised Aug. 6, 1992; accepted Aug. 10, 1992

We thank Beata Frydel and Julia O'Neal for histological support, Wes Eilbert for assistance with the experiments, and Phil Pfalzgraf for photography processing. We also thank Matthew Ennis and Heather J. Duncan for comments on the manuscript. This work was supported by DAMD 17-91-C-1071, NIDCD DC00347, and NINDS NS29218.

Correspondence should be addressed to Michael T. Shipley, Department of Anatomy and Cell Biology, ML 521, University of Cincinnati College of Medicine, 231 Bethesda Avenue, Cincinnati, OH 45267-0521.

Copyright (C) 1993 Society for Neuroscience $0270-6474 / 93 / 130650-10 \$ 05.00 / 0$ temporally in the olfactory system. Temporal coordination may be particularly important to olfactory circuit function, as this system lacks the point-to-point topographical organization characteristic of other sensory systems.

[Key words: brain, olfactory bulb, ACh, GABA, basal forebrain, anterior commissure]

The nucleus of the diagonal band (NDB) is a part of the magnocellular basal forebrain system which also includes the medial septum, which projects to the hippocampus, and nucleus basalis, which projects to neocortex (Shute and Lewis, 1967; Broadwell, 1975; Divac, 1975; Broadwell and Jacobowitz, 1976; Moyano and Molina, 1982; Mesulam et al., 1983; Sofroniew et al., 1987). NDB innervates the ipsilateral main olfactory bulb (MOB), primary olfactory cortex, the parahippocampal region, and the amygdala. Recent studies have revealed heterogeneity in transmitter content among these systems. In the progression from nucleus basalis, through medial septum to NDB, which represents a progression in target structures from neocortex to allocortex to paleocortex, there is a decreasing proportion of cholinergic neurons and an increasing proportion of GABAergic neurons (Rye et al., 1984; Wainer et al., 1985; Brashear et al., 1986; Zaborszky et al., 1986). In view of these differences in transmitter content, it is not clear whether the basal forebrain projections perform similar functions in the different target structures.

One proposed function of the medial septal projection is regulation of transmitter release from the terminals of commissural and association axons in hippocampus (Yamamoto and Kawai, 1967; Hounsgaard, 1978; Ben-Ari et al., 1981; Krnjevic, 1981; Valentino and Dingledine, 1981; Rovira et al., 1983). This presynaptic regulation appears to be mediated by $\mathrm{ACh}$. The anterior commissure $(\mathrm{AC})$ is analogous to the hippocampal commissure and the neocortical commissural system, the corpus callosum. The anterior wing of $\mathrm{AC}$ connects the two main olfactory bulbs. These AC fibers originate in the contralateral anterior olfactory nucleus (AON) and terminate predominantly in the internal plexiform (ipl) and granule cell ( $\mathrm{gcl}$ ) layers of the olfactory bulb, where they synapse with granule cells. The centrifugal projection from NDB terminates to some degree in all portions of the olfactory bulb; however, there is a particularly heavy termination in the internal plexiform layer and the superficial half of the gcl (Macrides et al., 1981; Luskin and Price, 1983; Macrides and Davis, 1983; Alheid et al., 1984).

Thus, a major part of the NDB projection to the bulb terminates in a location appropriate to contact commissural terminals. In view of the evidence for a presynaptic inhibitory action of $\mathrm{ACh}$ in hippocampus, it is possible that one action of 
the NDB terminals in the olfactory bulb is regulation of transmitter release from AC terminals. Therefore, we sought to determine if the projection from NDB regulates synaptic transmission in the anterior wing of the $\mathrm{AC}$ and whether $\mathrm{ACh}$ mediates this regulation.

\section{Materials and Methods}

Surgical preparation. Adult, male Sprague-Dawley rats were anesthetized with chloral hydrate $(400 \mathrm{mg} / \mathrm{kg}$ supplemented as needed) or with the gas anesthetic methoxyflurane (Metofane). Levels of both anesthetics were adjusted to maintain surgical anesthesia as judged by depth and rate of respiration and the presence of a moderate foot withdrawal reflex. Body temperature was maintained at $37^{\circ} \mathrm{C}$ with a heating pad. Anesthetized animals were placed in a stereotaxic apparatus tilted at a $30^{\circ}$ angle around the rostral-caudal axis, and the lateral aspect of the olfactory bulb and the lateral olfactory lract (LOT) were exposed by craniotomy after removing the contents of the orbit. The dorsal aspect of the skull was also exposed by a scalp incision.

Recording. Glass capillary pipettes ( $\sim 5 \mu \mathrm{m}$ tip diameter), filled with $1 \mathrm{M} \mathrm{NaCl}$, were used for most recordings. DC preamplification was used in all experiments; the amplified signal was usually band-pass filtered between $0.1 \mathrm{~Hz}$ and $7.0 \mathrm{kHz}$. A recording electrode was inserted into the olfactory bulb before insertion of any stimulation electrodes so that bulb responses could he used to guide placement of the stimulating electrodes. In all records presented here, the recording electrode was positioned in the granule cell layer (gcl), which was localized by physiological criteria (see below, Stimulation). Data acquisition, display, and control of stimulus timing were done by computer (Motorola VME/10). Amplitudes of field potential responses were measured from the baseline to the peak of the negative [AC and piriform cortex (PC)] or positive (LOT) deflection.

Stimulation. Coordinates for the placement of stimulation electrodes were determined from the stereotaxic atlas of Paxinos and Watson (1986). Monopolar stimulating electrodes (000 insect pins insulated except at the tip) were lowered stereotaxically into the contralateral anterior wing of the anterior commissure (AC). Optimal placement was determined by observation of field potentials in the bulb generated by stimulation through this AC electrode. After the AC was located, the stimulating electrode was cemented to the skull using $\alpha$-cyanoacrylate adhesive and dental acrylic. In some experiments, a second electrode of similar construction was placed into the posterior part of the ipsilateral piriform cortex $(\mathrm{PC})$ to stimulate ipsilateral afferents to the $\mathrm{MOB}$. This electrode was also cemented to the skull after optimal placement was determined. A concentric bipolar stimulating electrode $0.25 \mathrm{~mm}$ outer diameter, $0.75 \mathrm{~mm}$ tip separation; Rhodes Medical Instruments, Woodland Hills, CA), was guided stereotaxically into the NDB through a burr hole in the parietal bone. Optimal placement of this NDB electrode was determined by observation of the characteristics of the field potential generated by stimulating NDB (Nickell and Shipley, 1988).

A twisted bipolar stimulating electrode $(100 \mu \mathrm{m}$ wires, $300 \mu \mathrm{m}$ tip separation) was placed against the lateral olfactory tract (LOT) under visual guidance. This electrode was used to antidromically activate axons of the mitral cells, the principal output neurons of the olfactory bulb. Antidromic activation of the mitral cells produces a well-characterized field potential that undergoes a change of polarity at the mitral cell body layer (mcl) (Phillips et al., 1963). This transition and the presence of characteristic unit potentials were used to determine the location of the mcl. The location of the mcl thus served as an indicator of the depth of the recording electrode, which was then advanced into the gcl.

All stimulating currents were delivered by a constant current stimulus isolation unit. Stimulating currents for the $\mathrm{AC}$ and $\mathrm{PC}$ electrodes were adjusted to two to three times threshold. With the monopolar stimulating electrodes used, this required $200 \mu \mathrm{A}$ pulses of $100 \mu \mathrm{sec}$ duration. For the AC electrode located in the contralateral hemisphere, there is little danger of unintentional stimulation of structures projecting to the ipsilateral bulb. The stimulating electrode in posterior PC is likewise remote from other structures that project to MOB. Current spread could, however, cause antidromic activation of LOT, which runs along the surface of PC, or of LOT terminals in the superficial layer of PC. However, antidromic LOT activation produces a field potential of opposite polarity to that of the PC association inputs to MOB. Thus. we adjusted the position of the stimulation electrode and the stimulating current parameters to produce a stable $\mathrm{PC}$ field potential without contamination from LOT activation. Stimulation of NDB required somewhat higher currents $(300 \mu \mathrm{A}, 300 \mu \mathrm{sec})$ because of the use of a bipolar stimulating electrode and the large extent of the nucleus. Issues related to selective stimulation of this nucleus have been discussed previously (Nickell and Shipley, 1988). After each experiment, sufficient current was passed through the electrodes to produce lesions at the stimulation site; the locations of the lesions were later determined in histological sections.

Surface application of drugs. In some experiments, drugs were applied directly to the exposed surface of the olfactory bulb. Soman (pinacolylmethylphosphonofluoridate; $1 \mathrm{~mm}$ ) and atropine (10 mM) were dissolved in artificial cerebrospinal fluid (ACSF), and either drug was gently applied to the bulb surface with a $50 \mu 1$ syringe. The drug solutions were removed by wicking away the solution and washing with ACSF. In the case of the irreversible cholinesterase inhibitor soman, the extent of $\mathrm{AChE}$ inhibition at the termination of the experiment was determined by processing the tissue for AChE histochemistry (Van Ooteghem and Shipley, 1984).

Microinjection of drugs. In other experiments, drugs were injected directly into the olfactory bulb from the recording pipette. Recording pipettes of approximately $10 \mu \mathrm{m}$ tip diameter were filled with drug solutions (carbachol, ACh) in ACSF and injected by short pressure pulses (Picospritz). Injected volumes were estimated by measuring the diameter of the droplet created in air by a fixed number of pressure pulses. The volume of solution injected by this method was, however, somewhat uncertain because of the possibility of plugging of the pipette during insertion into the bulb. Thus, a method was developed that allowed direct measurement of injected volume and injection of an equal volume of vehicle into the same location.

Microbore pipettes (Fisher Scientific Co., catalog number $21-164-2 \mathrm{~A}$ ) were used. In these pipettes, ejected volumes of $60 \mathrm{nl}$ result in a meniscus movement of $1 \mathrm{~mm}$. To fabricate the double-barreled micropipette, one microbore tube was shortened and then bent in a flame. This shortened and bent tube was glued firmly to a second, straight microbore tube using epoxy cement. The straight, uncut, microbore tube was inserted into the chucks of a vertical pipette puller. The two tubes were then fused by heating them sufficiently to allow the chuck to be rotated onehalf turn in each direction. After cooling, the fused tubes were pulled at a normal heat setting. The resulting double-barreled pipette was broken under a microscope to about $30 \mu \mathrm{m}$ diameter. For recording evoked potentials, a length of Formvar-insulated stainless steel wire ( 2 mil, 50 $\mu \mathrm{m}$ ) was then brought into contact with the tip and fastened with a drop of cyanoacrylate adhesive. The wire was trimmed to extend about 200 $\mu \mathrm{m}$ past the end of the pipettes, leaving the tip uninsulated. Each microbore pipette was filled by applying suction while the pipette tip was immersed in the appropriate solution. Using these pipettes, drug or vehicle solutions were injected by short $(20 \mathrm{msec})$ pressure pulses $(\sim 10$ psi); the volume of fluid injected was measured by observing movement of the meniscus relative to a millimeter scale attached to the microbore tube.

Antidromic stimulation thresholds of AON terminals. Initial experiments suggested that inhibition of the AC field potential might be caused by presynaptic inhibition of AC terminals. Thus, we tested whether inhibition of the field potential was associated with changes in the antidromic activation threshold of $\mathrm{AC}$ terminals.

Terminals of AON neurons projecting to the ipsilateral bulb were activated using a bipolar stimulation and recording electrode constructed by gluing two Formvar-insulated stainless steel wires $(50 \mu \mathrm{m}$ diameter) side by side with $400 \mu \mathrm{m}$ tip separation. The deeper of these electrodes was also used to record AC and NDB field potentials. A small switch located near the preparation allowed connection to the amplificr or stimulator without disturbance of the electrodes. The electrode assembly was advanced until the forward tip was about $100 \mu \mathrm{m}$ below the mcl as determined by observation of changes in the LOT waveform.

Single units in the contralateral AON were recorded using tungsten microelectrodes. AON units driven by stimulation of the ipsilateral bulb were tested for antidromic activation by three tests: (1) constant latency, (2) ability to follow a train of three shocks at $3 \mathrm{msec}$ intervals, and (3) the occlusion of an antidromicically evoked spike by collision with a spontaneous discharge (collision test). Unit activity was recorded on instrumentation tape; peristimulus time histogram (PSTH) records were constructed on line using a computer.

\section{Results}

Field potential responses produced by AC stimulation. An extracellular electrode placed in the gcl of the olfactory bulb re- 


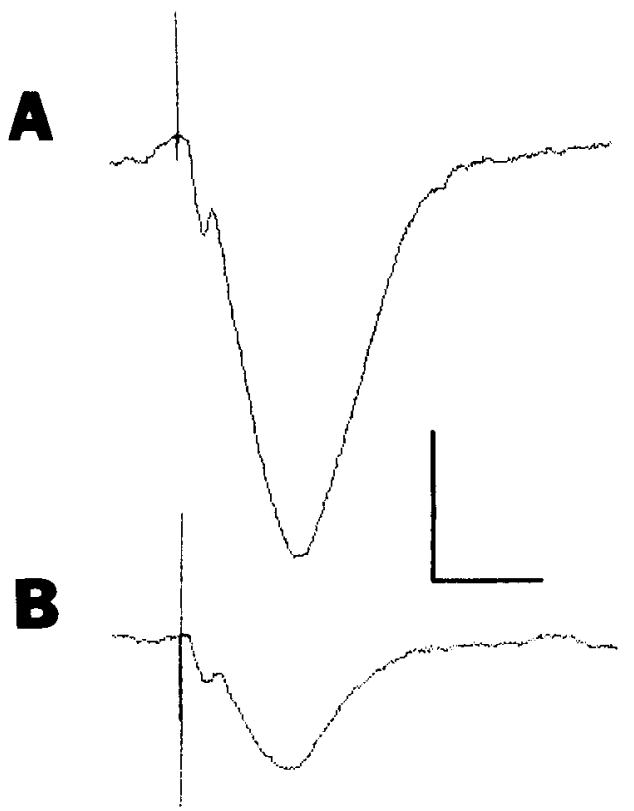

Figure 1. Inhibition of AC field potential by microinjection of carbachol into the olfactory bulb. $A$, Normal AC potential recorded by an clcctrode placed in the gcl. $B$, AC potential inhibited by injection of 5 $\mathrm{nmol}(500 \mathrm{nl}$ of $10 \mathrm{~mm})$ carbachol. Calibration: $1 \mathrm{mV}, 25 \mathrm{msec}$.

cords a negative-going field potential (Fig. 1 $A$ ) in response to AC stimulation (Walsh, 1959; Nakashima et al., 1978). The mechanisms responsible for this field potential have been well described. The field potential is the result of synchronous postsynaptic excitatory currents flowing into the granule cells in the gcl (Walsh, 1959; Yamamoto et al., 1963; Mori and Takagi, 1978; Nakashima et al., 1978). The extracellularly recorded ACevoked field potential is therefore a good index of the total synaptic current entering the population of granule cells after AC stimulation.

Effect of cholinergic agonists on the AC-evoked field potential. To test for cholinergic inhibition of this $\mathrm{AC}$ response, the cholinergic agonists carbachol $(10 \mathrm{mM}, n=5)$ or ACh $(1 \mathrm{mM}, n=$ 3) were locally injected into the ipl and gel of the olfactory bulb from the recording pipette. Injections (estimated at 100-500 nl over $10 \mathrm{~min}$ ) of either agonist consistently depressed the amplitude of the $\mathrm{AC}$ response (Fig. $1 B$ ). The depression caused by $A C h$ reversed after about $30 \mathrm{~min}$, whereas the depression caused by carbachol did not reverse within the period of observation ( $>1 \mathrm{hr}$ ). Saline or ACSF injections of similar volume produced only small and transient changes in the amplitude of the $\mathrm{AC}$ response.

Because drug delivery from the recording pipette did not allow precise measurement of the volumes injected, we conducted additional experiments using a calibrated double-barreled pipette (Materials and Methods). In addition, since the AC synaptic response exhibits significant facilitation with repeated shocks (Mori and Takagi, 1978), we tested with trains of 4-10 AC shocks to determine if inhibition caused by carbachol injections could be reversed by homosynaptic facilitation.

Injections of $5 \mathrm{nmol}$ carbachol solution (100 $\mathrm{nl}$ of $50 \mathrm{~mm}$ carbachol in saline or ACSF) caused a 50\% decrease in the amplitude of the AC response. Systemic (i.p.) injection of $1 \mathrm{mg}$ of scopolamine, however, reversed the carbachol inhibition within $5 \mathrm{~min}$. In the same preparations, equal or larger volumes of vehicle ejected from the second barrel caused only small changes in response amplitude $(n=4)$. These changes are illustrated in Figure 2, which shows the amplitudes of the field potential responses to a train of $10 \mathrm{AC}$ shocks, given at 100 msec intervals. As indicated in the figure caption, the data presented were recorded before and after a microinjection of 200 $\mathrm{nl}$ of saline, after microinjection of $100 \mathrm{nl}(5 \mathrm{nmol})$ carbachol, and after systemic injection of $1 \mathrm{mg}$ of scopolamine. The total volume of vehicle or drug solution was injected over a period of $5 \mathrm{~min}$. The decrease in response amplitude caused by drug injection continued for an additional $10 \mathrm{~min}$. With repeated shocks, there is moderate facilitation of the AC response amplitude, but the response did not return to control amplitude.

Site of carbachol action. The late positive-going component of the field potential caused by stimulation of the LOT, like the AC-evoked field potential, is the result of synaptic currents flowing into granule cells (Rall and Shepherd, 1968). A lack of changes in the LOT field potential after injection of carbachol would therefore be evidence that inhibition of the $\mathrm{AC}$ response is not caused by shunting or depolarization of the granule cells. Thus, the amplitude of the antidromic LOT field potential was measured to determine whether carbachol injection also depressed the LOT antidromic field potential. Carbachol injections produced no measurable effect on the amplitude of the LOT potential (Fig. 2B). Thus, the site of action of carbachol is unlikely to be a depolarization or shunting of the granule cells.

Effect of NDB stimulation and AChE blockade on the $A C$ response. The inhibition of the $\mathrm{AC}$ response by cholinergic agonists suggested that endogenously released $\mathrm{ACh}$ inhibits synaptic transmission in the commissural pathway. If this is true, then electrical stimulation of NDB should release $\mathrm{ACh}$ and re-
Figure 2. Comparison of the effects of saline or carbachol injections on the amplitude of $\mathrm{AC}$ and LOT field potentials in the olfactory bulb. $A$, AC responses: amplitudes of 10 successive field potentials recorded in the $\mathrm{gcl}$ of the olfactory bulb in response to a 10 $\mathrm{Hz}$ train applied to $\mathrm{AC} . B$, LOT responses: amplitudes of responses to a similar train of shocks applied to LOT. These graphs compare response amplitudes before and after intrabulbar injection of $200 \mathrm{nl}$ of ACSF or $5 \mathrm{~nm}$ carbachol in $100 \mathrm{nl}$ of ACSF, or after systemic injection of $1 \mathrm{mg}$ of scopolamine.
A

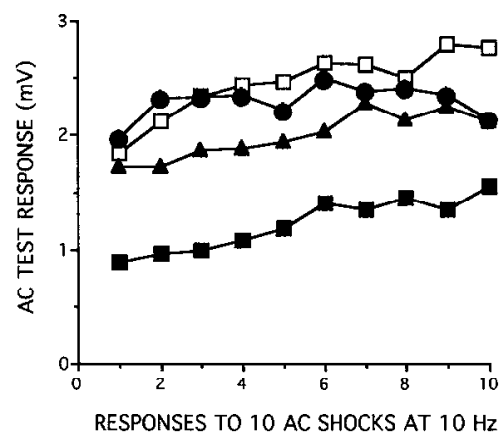

B

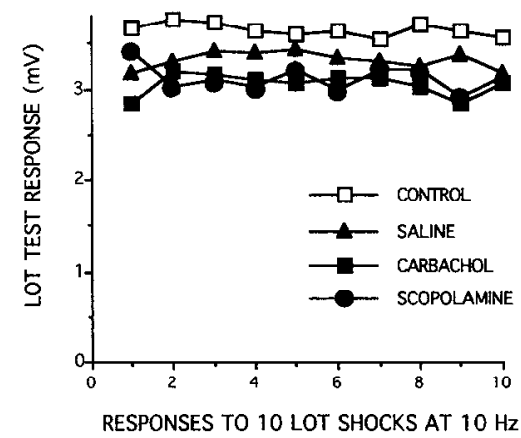




\section{NORMAL AChE}

A

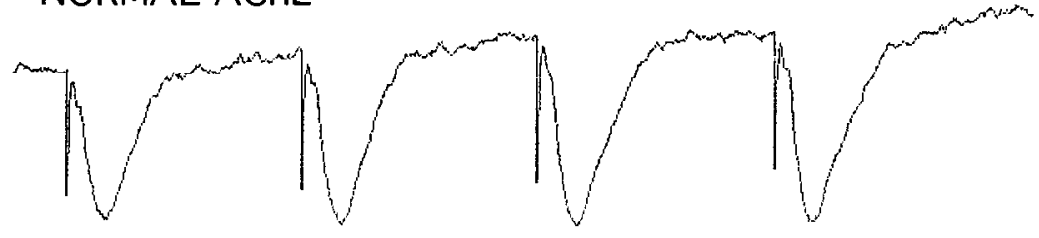

B
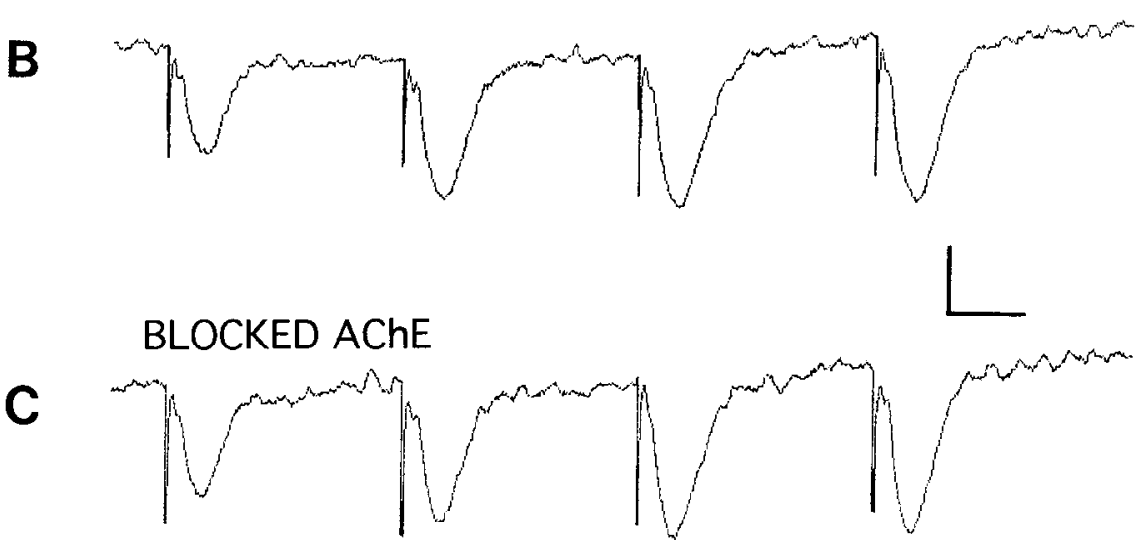

D
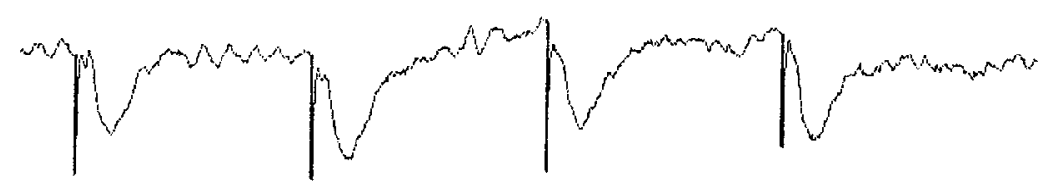

Figure 3. Effect of NDB stimulation on AC responses before and after cholinesterase inhibition. $A$, Field potentials recorded in the gcl of the olfactory bulb in response to four shocks applied to $\mathrm{AC}$. $B$, Four responses to $\mathrm{AC}$ shocks beginning $500 \mathrm{msec}$ after termination of a train of $4010 \mathrm{~Hz}$ shocks applied to NDB. Note that the initial responses to $\mathrm{AC}$ stimulation are reduced by prior stimulation of NDB. $C$, Following local inhibition of $\mathrm{AChE}$ in the olfactory bulb, the initial responses to $\mathrm{AC}$ stimulation are reduced to a similar extent as following NDB stimulation $(B)$. Response amplitudes increase with successive $A C$ shocks. $D$, Effect of NDB conditioning shocks (as in $B$ ) on responses to $A C$ stimulation following inhibition of $\mathrm{AChE}$ (as in C) Initial responses are further suppressed; in contrast to $C$, there is little facilitation with repeated shocks to AC. Note: Oscillatory responses seen in these records are $50 \mathrm{~Hz}$ spontaneous activity often present after NDB stimulation. Calibration: $1 \mathrm{mV}, 50 \mathrm{msec}$. duce the AC response amplitude. Similarly, blockade of the degradative enzyme for $\mathrm{ACh}, \mathrm{AChE}$, in the bulb might cause buildup of $\mathrm{ACh}$ tonically released from NDB terminals; this should also inhibit the $A C$ response. Thus, we investigated the effects of NDB stimulation and of local AChE inhibition on the amplitude of the AC-evoked field potential.

Single or double NDB shocks did not cause any measurable inhibition of the $\mathrm{AC}$ field potential (not illustrated). We therefore tested the effect of short trains of NDB shocks on the AC response amplitude $(n=5)$. Typical results of these tests are shown in Figure $3, A$ and $B$, and Figure 4 . Figure $3 B$ shows field potential responses to a train of four $A C$ shocks beginning 500 msec after termination of 40 NDB shocks. As shown, the initial AC response is inhibited by the NDB stimulation, but the amplitude increases with repeated $\mathrm{AC}$ shocks so that the fourth shock is approximately the same amplitude as the control. Increasing the number of NDB shocks from 40 to 120 caused only a small additional inhibition of the $\mathrm{AC}$ response (Fig. 4). In Figure 4 , the amplitudes of the first $(A)$ and the fourth $(B) \mathrm{AC}$ responses are plotted. Because of facilitation of the $\mathrm{AC}$ response, the amplitude of the fourth $\mathrm{AC}$ response is always larger than the control amplitude of the first response. Thus, the inhibition caused by NDB stimulation can be partially overcome by facilitation of the AC pathway.

To determine the effect of AChE inhibition, we applied the irreversible cholinesterase inhibitor soman to the exposed surface of the olfactory bulb (1 $\mathrm{mm}$ in saline; see Materials and Methods). Because the enzyme inhibition caused by soman is irreversible, the region of the bulb in which $\mathrm{AChE}$ was inhibited could be determined by processing the tissue for AChE histochemistry subsequent to the electrophysiological experiment. Soman caused a well-defined zone of AChE inhibition, with complete inhibition on the lateral side of the bulb and normal AChE activity elsewhere (Fig. 5).

In all cases $(n=10)$, AChE inhibition decreased the $\mathrm{AC}$ response. The $\mathrm{AC}$ response amplitude decreased gradually for 20-30 min after application of soman, presumably reflecting the spread of AChE inhibition and the accumulation of tonically
A

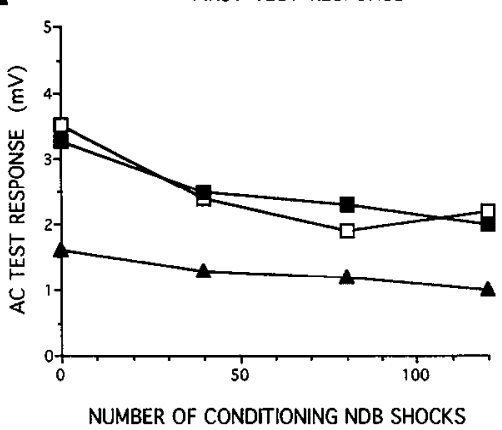

B

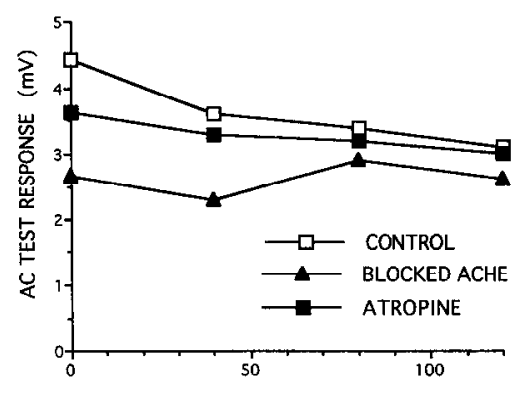

NUMBER OF CONDITIONING NDB SHOCKS
Figure 4. Effects of AChE inhibition and muscarinic receptor antagonism on NDB-induced inhibition of AC field potentials. $A$, Response amplitude to the first of four AC shocks before and after AChE inhibition. Application of the muscarinic antagonist, atropine restored the response amplitude to the same level measured prior to $\mathrm{AChE}$ inhibition. $B$, Amplitude of the response to the fourth of four AC shocks after $\mathrm{AChE}$ inhibition was reduced less than the amplitude of the first response $(A)$. Application of atropine restored the response amplitude to the same level measured prior to AChE inhibition. 
Figure 5. AChE inhibition following topical application of an irreversible cholinesterase inhibitor. A $1 \mathrm{~mm}$ solution of soman in saline was applied to the exposed lateral surface of the olfactory bulb. AChE was completely inhibited in the lateral side of the bulb from which the recordings of Figures 3 and 4 were made. The contralateral bulb (right side in micrograph) shows the normal distribution of AChE. Abbreviations: $E P L$, external plexiform layer; $G C L$, granule cell layer; $G L$, glomerular layer; $O N L$, olfactory nerve layer.

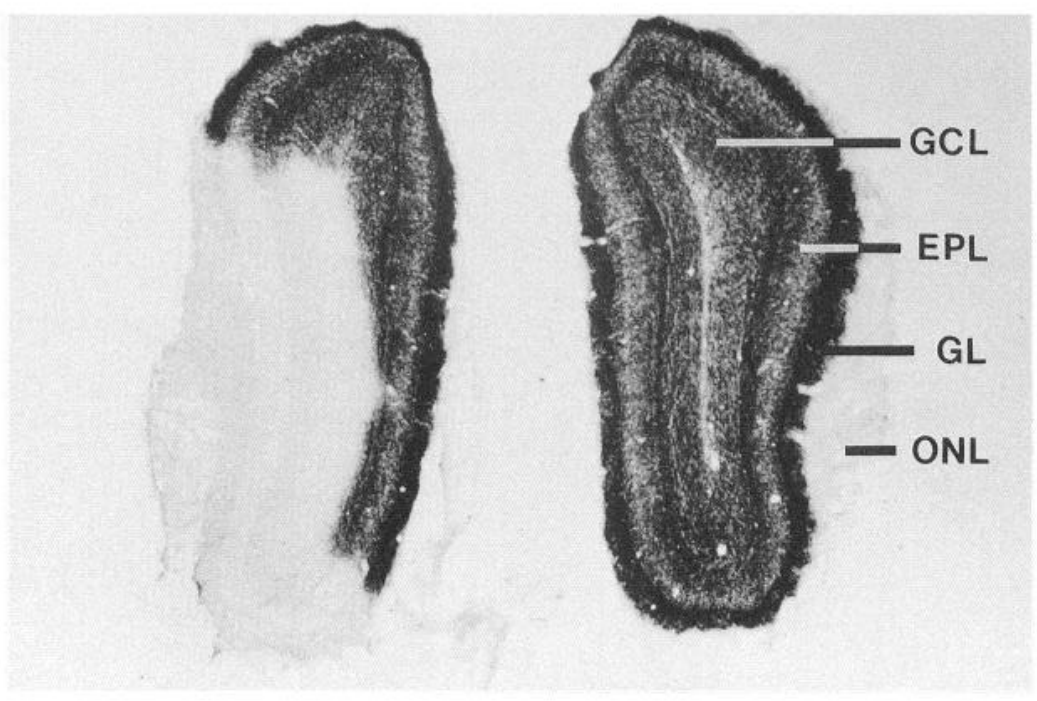

released $\mathrm{ACh}$. After $\mathrm{AChE}$ inhibition, NDB stimulation further reduced the amplitude of the AC potential. These effects are illustrated in Figure 3, $C$ and $D$, and Figure 4.

The reduction of the AC response by cholinesterase inhibition was reversed by topical application of $10 \mathrm{~mm}$ atropine in three of five cases tested (Fig. 4). In the remaining two cases, there was no recovery of response. In the cases in which atropine reversed the effects of $\mathrm{AChE}$ inhibition, NDB stimulation still inhibited the AC response (Fig. 4). This residual inhibition may result from a noncholinergic component of the NDB projection or to failure of the topically applied atropine to block all muscarinic receptors.

Profound, long-term inhibition of AC response by longer trains of $N D B$ shocks. We previously described the characteristics of the NDB-evoked field potential in MOB and its potentiation by $\sim 10 \mathrm{~Hz}$ stimulation (Nickell and Shipley, 1988). This stimulation rate is similar to the normal firing rate of NDB neurons and to the theta rhythm, which may be regulated by basal forebrain cholinergic neurons (Andersen et al., 1979; Macrides et al., 1982). Single NDB shocks produce a biphasic field potential

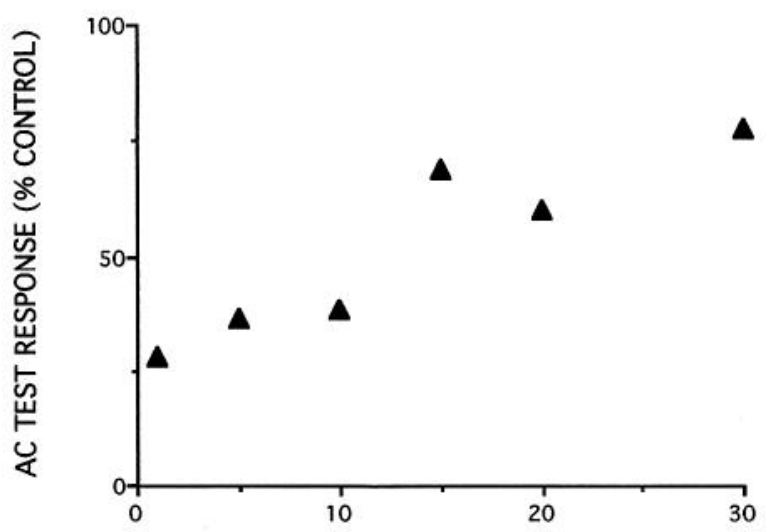

TIME AFTER NDB STIMULATION (SEC)

Figure 6. Duration of inhibition of AC field potential responses caused by NDB stimulation. NDB was stimulated by 160 shocks at $10 \mathrm{~Hz}$ and $\mathrm{AC}$ was tested with a single shock to $\mathrm{AC}$ at the intervals indicated. The inhibition has a time course of approximately $30 \mathrm{sec}$. There was a 10 min rest period between trials. recorded in the gcl of the olfactory bulb. Stimulation for several seconds at $10 \mathrm{~Hz}$ causes a marked potentiation of this field potential, which decays to half-amplitude within 2 sec after termination of the stimulating train. Thus, $10 \mathrm{~Hz}$ NDB stimulation causes pronounced and relatively long-lasting changes in the state of the olfactory bulb circuitry that are not apparent with single or short trains of NDB shocks.

In contrast to the moderate inhibition of the $\mathrm{AC}$ response caused by shorter trains of NDB shocks, trains of NDB stimulus shocks sufficient to cause potentiation of the NDB field potential caused a profound and long-lasting inhibition of the $\mathrm{AC}$ response ( $n=20$ animals). In some preparations, the $\mathrm{AC}$ response was completely suppressed for many seconds after NDB stimulation. The duration of the inhibition was determined by measuring the amplitude of responses to single test AC shocks delivered at different intervals after NDB stimulation (Fig. 6). A $10 \mathrm{~min}$ rest period was allowed between each trial and a control AC response was obtained immediately before each period of NDB stimulation. The response returns to $50 \%$ amplitude after $11 \mathrm{sec}$ and is $75 \%$ of control after $30 \mathrm{sec}$.

Figure 7 shows the response to $\mathrm{AC}$ stimulation before and after NDB stimulation in two separate experiments. In both experiments, the initial AC response is completely suppressed at $10 \mathrm{sec}$ (Fig. $7 \mathrm{~A}$ ) and $3 \mathrm{sec}$ (Fig. $7 \mathrm{~B}$ ) after a train of $160 \mathrm{NDB}$ shocks. With repeated AC shocks, however, the AC response amplitude rapidly increases. In each preparation, the response to a single AC shock would have been inhibited for many additional seconds in the absence of repeated AC stimulation. Thus, the increase in response amplitude seen in Figure 7 is caused by facilitation of the $\mathrm{AC}$ response and not by passive decay of the inhibition.

Effect of NDB stimulation on other synaptic inputs to granule cells. NDB stimulation sufficient to cause prolonged inhibition of the $\mathrm{AC}$ response produces a shorter-lasting potentiation of the NDB field potential. The LOT response is not significantly affected by such stimulation (Nickell and Shipley, 1988). Like the AC field potential, the NDB and the LOT field potentials result from synaptic currents flowing into granule cells (Rall and Shepherd, 1968; Nickell and Shipley, 1988), although the LOT potential is the result of synaptic activity in the more superficial gcl. Thus, the absence of inhibition of NDB and LOT responses after NDB stimulation suggests that the NDB-induced inhibi- 
tion of the $\mathrm{AC}$ response is not the result of changes in granule cells. This was further assessed by determining the effect of NDB stimulation on the field potential evoked in the olfactory bulb by stimulation of ipsilateral PC. This projection terminates on granule cells in a pattern similar to the termination of the $\mathrm{AC}$ (Davis and Macrides, 1981; Luskin and Price, 1983). Thus, if the inhibition of the AC field potential is caused by depolarization or shunting of granulc cells, then the PC potential should also be similarly inhibited.

NDB stimulation sufficient to cause potentiation of the NDB field potential and inhibition of the AC field potential causes a potentiation of the $\mathrm{PC}$ field response (Fig. $8 A$ ). Like the potentiated NDB field potential, the PC response returned to control amplitude within $2 \mathrm{sec}$ after the end of NDB stimulation (Fig. $8 B$ ). In contrast with the potentiation of the $\mathrm{PC}$ response, the $A C$ field potential was, as in previous experiments, inhibited at all times after NDB stimulation (Fig. 8B,C). Identical results were obtained when the order of the $\mathrm{AC}$ and PC shocks was reversed. At longer periods after NDB stimulation, both $\mathrm{AC}$ and $P C$ responses were inhibited. Results similar to those illustrated in Figure 8 were obtained in five preparations. Thus, the PC response is increased for $\sim 2 \mathrm{sec}$ after NDB stimulation, at the same time that the $\mathrm{AC}$ response is profoundly depressed. Since all these responses (LOT, NDB, PC) result from synaptic currents flowing into granule cells, it is improbable that the $\mathrm{AC}$ inhibition results from depolarization or shunting of the granule cells. This suggests, therefore, that an alternative site of the inhibition of the AC response by NDB stimulation is a modification of the AC terminals. Facilitation of the inhibited AC response by repeated AC shocks (homosynaptic facilitation), as described above, is also consistent with changes in the AC presynaptic terminals. These considerations suggested that inhibition of the AC response is caused by reduced transmitter release from $A C$ terminals rather than by postsynaptic mechanisms such as depolarization or shunting of the target granule cells. Thus, we tested directly whether NDB stimulation causes a change in the excitability of AC terminals.

$N D B$ stimulation decreases the excitability of $A C$ terminals. Presynaptic inhibition of transmitter release by spinal nerves is accompanied by depolarization and an increase in excitability of the nerve terminals (Wall, 1958; Eccles et al., 1961, 1962a,b, 1963). Thus, we tested whether the decrease in the AC field potential amplitude caused by NDB stimulation is accompanied by a change in the excitability of AC terminals in the olfactory bulb.

A bipolar stimulating and recording electrode (Materials and Methods) was placed in the olfactory bulb with the leading tip located in the superficial part of the gcl 100-200 $\mu \mathrm{m}$ below the $\mathrm{mcl}$. The effect of NDB stimulation on the amplitude of the AC field potential was observed by recording through the leading tip of this bipolar electrode. NDB stimulation parameters were adjusted to produce the prolonged inhibition of the $\mathrm{AC}$ response illustrated in Figure 6.

Contralateral AON neurons activated by stimulation of the AC were isolated by slowly advancing an extracellular recording electrode through the AON while periodically stimulating through the AC electrode. Units driven by AC stimulation were tested for antidromic activation from the ipsilateral olfactory bulb electrode using the tests of constant latency, high-frequency following, and the collision test (Materials and Methods). Six of 12 antidromically activated units exhibited a latency of 3-6 msec; six other units that passed all three tests for antidromic
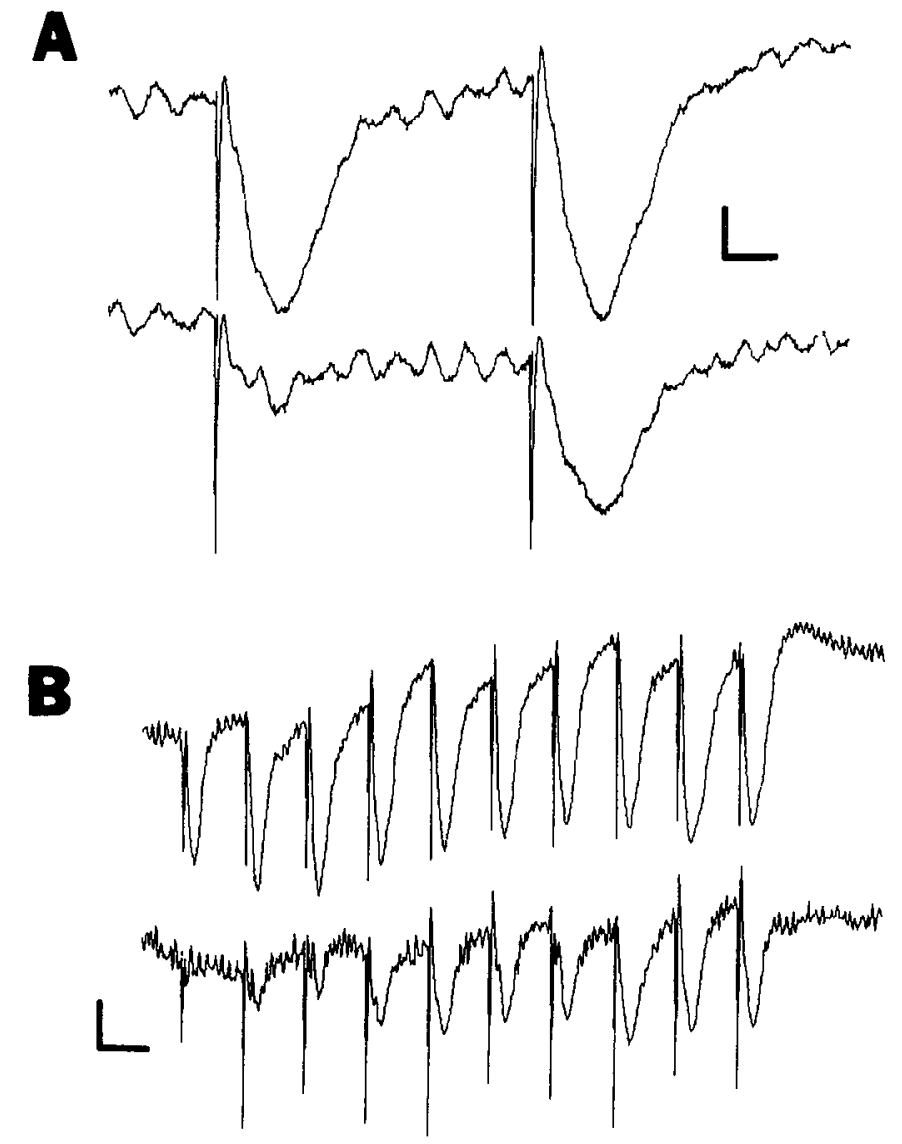

Figure 7. Repetitive stimulation of AC reduces the duration of NDBinduced inhibition. $A$, Field potential responses to two AC test shocks $3 \mathrm{sec}$ after termination of a train of 160 NDB shocks. $B$, Field potential responses to $10 \mathrm{AC}$ test shocks $10 \mathrm{sec}$ after termination of a train of 160 NDB shocks. At both delay intervals, the initial AC responses are profoundly inhibited. However, with repeated shocks to AC, the responses approach control values in a much shorter time than without facilitation (see Fig. 6). (Note that oscillatory responses seen in these records are $50 \mathrm{~Hz}$ spontaneous activity often present after NDB stimulation.) Calibration: $A, 1.0 \mathrm{mV}, 20 \mathrm{msec} ; B, 1.0 \mathrm{mV}, 130 \mathrm{msec}$.

activation had much longer latencies between 8 and $16 \mathrm{msec}$. These long-latency responses may represent neurons activated through small terminal branches of the AON axons.

The probability of antidromically activating the isolated AON neuron was determined by constructing a PSTH of the neurons' antidromic responses to 20 bulb shocks given at $300 \mathrm{msec}$ intervals. So that changes in excitability could be detected, stimulus current through the bulb electrode was critically adjusted to be near threshold. Initial tests indicated that the excitability of AON terminals was decreased following NDB stimulation. No units were found for which the excitability was increased. Thus, in subsequent experiments the stimulus strength applied to AC terminals was adjusted so that $18-20$ of 20 stimulus shocks produced a spike in the isolated contralateral AON neuron.

Using these parameters, we tested for changes in the antidromic activation threshold of the isolated AON unit following NDB stimulation. Construction of the PSTH for antidromic activation of each AON unit began $500 \mathrm{msec}$ after termination of the NDB stimulation. The threshold was considered changed only if the number of antidromic spikes decreased by at least 

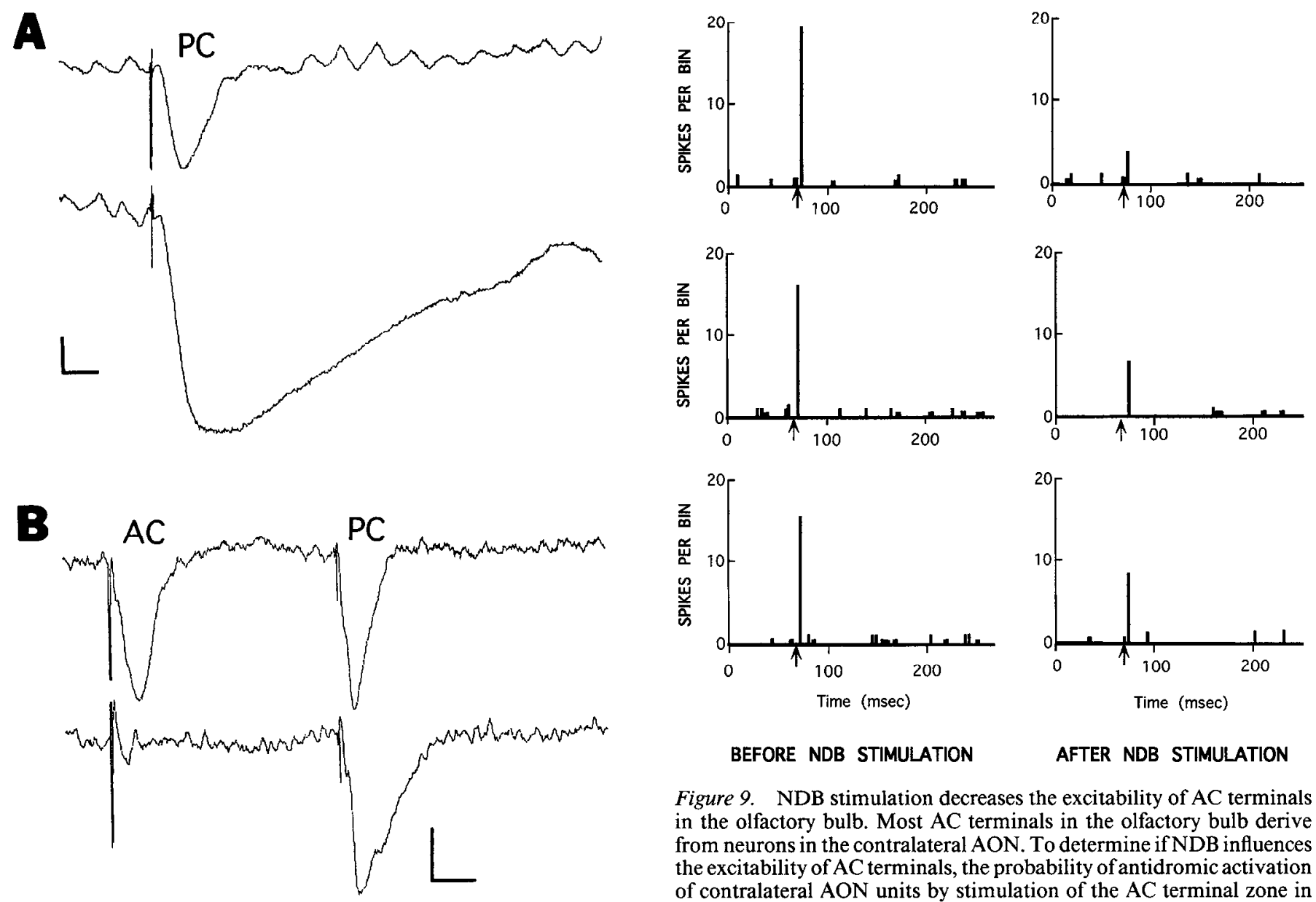

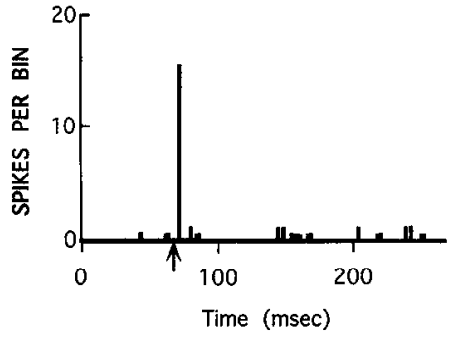

BEFORE NDB STIMULATION

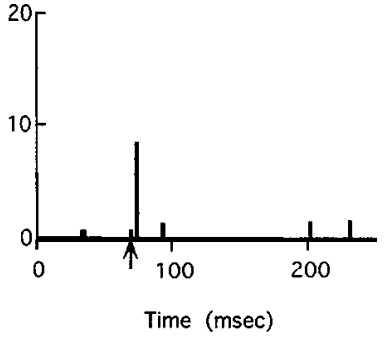

AFTER NDB STIMULATION

Figure 9. NDB stimulation decreases the excitability of AC terminals in the olfactory bulb. Most AC terminals in the olfactory bulb derive from neurons in the contralateral $\mathrm{AON}$. To determine if NDB influences the excitability of AC terminals, the probability of antidromic activation of contralateral AON units by stimulation of the AC terminal zone in the ipsilateral olfactory bulb was measured before and after stimulation of NDB. Each pair of PSTH records shows the number of antidromic responses of a contralateral AON neuron to 20 shocks to the olfactory bulb before (left) and after (right) 160 shocks $(10 \mathrm{~Hz})$ to NDB. Time of antidromic test shock is shown by arrows. Trials were separated by 8 min rests between NDB stimulation periods. The three pairs of PSTH records represent three independent replications of the same test on the same AON unit. Of 11 different AON units thus tested, 6 showed the illustrated NDB-induced decrease in terminal excitability.

$30 \%$ on each of three successive trials. Rest periods of $10 \mathrm{~min}$ were allowed between successive trials.

The effect of NDB stimulation was tested on the thresholds of 11 antidromically driven units in five animals. Of these 11 units, the excitability of 6 was decreased by NDB stimulation. This is illustrated in Figure 9, which shows a series of three pairs of PSTH records obtained before and after NDB stimulation. There is a consistent large decrease in the number of antidromic spikes following NDB stimulation. After NDB stimulation, most of the counted antidromic spikes occurred near the end of the 20 test shocks to AC, presumably because of decay of the inhibitory effect.

For each of the six neurons that passed the formal test for a change in activation threshold, a reduction of $50 \%$ or greater in the number of antidromic spikes could be obtained with critical adjustment of the stimulus strength. The remaining five units profoundly inhibited while the PC response is still slightly larger than control. $C$, Quantitative analysis of the time course of the differential effect of NDB stimulation on $A C$ and $P C$ responses. The experiment shown in $B$ was repeated, varying the time interval between cessation of the NDB conditioning shock train and the application of test shocks to $\mathrm{AC}$ and $\mathrm{PC}$. This analysis demonstrates that while the $\mathrm{AC}$ response is always inhibited following NDB stimulation, the $\mathrm{PC}$ response is initially potentiated, and then inhibited. The inhibitory phase of the $\mathrm{PC}$

response has a time course similar to that of the inhibition of the $\mathrm{AC}$ response. Calibration $(A$ and $B$ ): $1.0 \mathrm{mV}, 50 \mathrm{msec}$. 
were not consistently affected by NDB stimulation. There were no significant differences in the antidromic latencies of affected versus unaffected $A O N$ neurons. Thus, the prolonged inhibition of the AC response caused by NDB stimulation is accompanied by significant changes in excitability of AC terminals in the bulb, although the direction of the change is opposite that of spinal afferents.

\section{Discussion}

The results of the present experiments can be summarized as follows. (1) Exogenous application of cholinergic agonists reduces the amplitude of field potentials caused by electrical stimulation of AC. (2) Blockade of the ACh degradative enzyme $\mathrm{AChE}$ caused a similar depression of the AC response. (3) Both of these effects were blocked by muscarinic antagonists. (4) Stimulation of magnocellular basal forebrain neurons, which are the source of cholinergic input to the olfactory bulb, similarly inhibited the AC response. (5) The inhibition is selective to the $\mathrm{AC}$ response, as the responses evoked by stimulation of other afferents that synapse with granule cells were not inhibited by NDB stimulation. (6) The inhibition of the AC response was prolonged but could be rapidly reversed by homosynaptic facilitation. (7) The excitability of AC terminals in the olfactory bulb was significantly decreased by NDB stimulation.

Transmitters in the NDB projection. At least $25 \%$ of the NDB neurons projecting to the olfactory bulb are cholinergic (Rye et al., 1984; Wainer et al., 1985; Brashear et al., 1986; Zaborszky et al., 1986). The observed inhibition of the AC response after AChE inhibition in the olfactory bulb is therefore presumably due to buildup of ACh tonically released from these cholinergic terminals. Microinjection of cholinergic agonists into the vicinity of AC terminals in the bulb mimicked both the effect of AChE inhibition and NDB stimulation. The reversal of the inhibitory effects of both cholinergic agonists and AChE inhibition by muscarinic antagonists further supports the cholinergic nature of the inhibition and demonstrates the involvement of muscarinic receptors. Many other NDB neurons projecting to the olfactory bulb contain GABA (Rye et al., 1984; Wainer et al., 1985; Brashear et al., 1986; Zaborszky et al., 1986), and a peptide transmitter (galanin) has been colocalized with $\mathrm{ACh}$ in some NDB neurons (Melander et al., 1985). Electrical stimulation in NDB probably releases these transmitters and possibly others not yet identified. Thus, ACh, acting at muscarinic receptors, certainly contributes to inhibition of the $A C$ response by NDB stimulation, but other transmitters and receptors may also be involved.

Presynaptic locus of the inhibition. The inhibition of the AC response by cholincrgic agonists and by NDB stimulation is probably due to decreased transmitter release from AC terminals, that is, presynaptic inhibition. The evidence for this interpretation is as follows.

First, other synaptic inputs to granule cells are not similarly affected by NDB stimulation, cholinergic agonists, or AChE inhibition. The LOT potential, which is generated by currents flowing into granule cells from synapses located in the external plexiform layer and gcl, was not affected by any of the manipulations used in the present experiments. The ipsilateral PC field potentials, which are also generated by synaptic currents flowing into granule cells in the gcl, were strongly potentiated at short times after NDB stimulation, when the AC response was completely suppressed. The termination pattern of the ipsilateral $\mathrm{PC}$ projection in the bulb is similar to that of $\mathrm{AC}$ (Davis and
Macrides, 1981; Luskin and Price, 1983; Schoenfeld and Macrides, 1984). Therefore, unless NDB selectively terminates upon only those granule cell processes that also receive $\mathrm{AC}$ terminal input, the inhibition of the $\mathrm{AC}$ response cannot be the result of depolarization or shunting of granule cell membranes.

Second, the decreased excitability of AC terminals in the ipsilateral bulb following NDB stimulation is direct evidence for a presynaptic locus of the inhibition. In other systems, several presynaptic mechanisms have been implicated in modulation of transmitter release. Stimulation of spinal roots is followed by reduced transmitter release, depolarization of the nerve trunk, and increased excitability of terminals (Wall, 1958; Eccles et al., $1961,1962 \mathrm{a}, \mathrm{b}, 1963)$. Hounsgaard (1978) found increased terminal excitability following application of ACh to afferent fibers in the hippocampal slice. Presynaptic inhibition of glutamate release from crustacean excitatory motor axons is mediated by increased $\mathrm{Cl}^{-}$conductance (Takeuchi and Takeuchi, 1966). There is also evidence for increased $\mathrm{K}^{+}$conductance or blockage of voltage-gated $\mathrm{Ca}^{2+}$ channels as mediators of presynaptic inhibition (Dolphin and Scott, 1987; Bean, 1989). Any of these mechanisms might change the activation threshold of terminals.

The decreased excitability of AC terminals in the bulb contrasts with the increased excitability observed in spinal roots and hippocampus. This may indicate that the presynaptic inhibition described here is mediated by a different mechanism than mediates presynaptic inhibition in the hippocampus and spinal cord. Either an increase in $\mathrm{Cl}^{-}$conductance or a decrease in the voltage-sensitive $\mathrm{Ca}^{2+}$ conductance might reduce terminal excitability. However, regardless of the ionic mechanism, the fact that terminal excitability changes following NDB stimulation indicates that there is a direct effect of NDB stimulation on these terminals. This, together with the other characteristics of the inhibition of the AC response discussed above, suggests a presynaptic locus for the effect.

Comparison with analogous systems. The NDB projection to the olfactory bulb is analogous to the medial septal projection to the hippocampus. Both are part of the basal forebrain system that projects to cortical structures; both are partially cholinergic, although the medial septal projection contains a larger proportion of cholinergic fibers (Rye et al., 1984; Wainer et al., 1985; Brashear et al., 1986; Zaborszky et al., 1986). It is therefore of interest to compare present findings with previous studies of the medial septal-hippocampal system. As in the olfactory bulb, exogenous application of cholinergic agonists reduces transmitter release from terminals of commissural afferents in hippocampus (Yamamoto and Kawai, 1967; Hounsgaard, 1978; Krnjevic et al., 1981; Valentino and Dingledine, 1981; Krnjevic and Ropert, 1982; Rovira et al., 1983). Medial septal stimulation, however, increases the response of hippocampal granule cells to afferent inputs (Krnjevic and Ropert, 1982). Thus, the present study is apparently the first to demonstrate that inhibition of commissural terminals by exogenously applied $\mathrm{ACh}$ is mimicked by stimulation of the magnocellular basal forebrain neurons that are the source of endogenously released ACh.

A partial explanation for the difference between the effects of medial septum and NDB stimulation is that exogenously applied ACh excites pyramidal cells (Ben-Ari et al., 1981; Krnjevic et al., 1981; Krnjevic and Ropert, 1982; Cole and Nicoll, 1984), while the mitral cells of the olfactory bulb are not directly excited by cholinergic agonists or by NDB stimulation (McLennan, 1971; Nickell and Shipley, 1988). Therefore, the inhibitory actions of NDB stimulation on commissural terminals in the bulb may be manifest because it is not masked by excitation of mitral cells, 
whereas with electrical stimulation of the medial septum the excitatory actions of $\mathrm{ACh}$ on the pyramidal cells obscure the inhibitory actions of $\mathrm{ACh}$ on commissural and association inputs. It is possible that the characteristics of the inhibition of the AC terminals following NDB stimulation are similar to those that would be observed in commissural inputs to the hippocampus if the excitatory effects of septal stimulation on pyramidal cells were removed. Therefore, inhibition of afferent terminals may be a common feature of basal forebrain projections to cortical structures, although the proportion of the inhibition resulting from cholinergic synapses may differ.

Functional significance. Previous studies suggest that "information" sufficient to identify odors is transferred through the AC. Transection of the AC reduces olfactory acuity (Bennett, 1968). The AC is required for retrieval of olfactory memories from the opposite hemisphere (Teitelbaum, 1971; Kucharski et al., 1986; Kucharski and Hall, 1987). The profound AC response inhibition produced by NDB stimulation might be similar to a transection of the commissure: sufficient activation of NDB could effectively uncouple the two olfactory bulbs. The function of the cholinergic regulation of $\mathrm{AC}$ terminals may therefore be to regulate the transfer of information between the two bulbs.

More specific conjecture about the functional significance of NDB inhibition of AC terminals is limited by a lack of information about NDB. The NDB to MOB projection is analogous to the medial septum to hippocampus projection. The medial septum to hippocampus circuit provides the "pacemaker" for the hippocampal theta rhythm. Theta rhythm is also expressed in the olfactory bulb, especially during alert, exploratory sniffing. This rhythm has been shown to be phase locked with the sniff cycle and hippocampal theta during odor learning tasks (Macrides et al., 1982). Based on its anatomical similarities to the medial septum, it is reasonable to speculate that NDB is the pacemaker for bulb theta. Thus, it is possible that during alerted sniffing, NDB may phasically inhibit the terminals of contralateral $(A C)$ and ipsilateral (PC) feedback projections to the olfactory bulb. Such phasic modulation could function to coordinate the transfer of olfactory signals temporally to higherorder olfactory structures. Temporal coordination of circuit function may be critical to the olfactory system, which lacks the point-to-point spatial organization characteristic of other sensory systems (Shipley and Reyes, 1991; Nickell and Shipley, 1992).

\section{References}

Alheid GF, Carlsen J, DeOlmos J, Heimer L (1984) Quantitative determination of collateral anterior olfactory nucleus projections using a fluorescent tracer with an algebraic solution to the problem of double retrograde labeling. Brain Res 292:17-22.

Andersen P, Bland HB, Myrher T, Schwartzkroin PA (1979) Septohippocampal pathway necessary for dentate theta production. Brain Res 165:13-22.

Bean BP (1989) Neurotransmitter inhibition of neuronal calcium currents by changes in channel voltage dependence. Nature 340:153156.

Ben-Ari Y, Krnjevic K, Reinhardt W, Ropert N (1981) Intracellular observations on the disinhibitory action of acetylcholine in the hippocampus. Neuroscience 6:2475-2484.

Bennett MH (1968) The role of the anterior limb of the anterior commissure in olfaction. Physiol Behav 3:507-515.

Brashear HR, Zaborszky L, Heimer L (1986) Distribution of GABAergic and cholinergic neurons in the rat diagonal band. Neuroscience 17:439-451.

Broadwell RD (1975) Olfactory relationships of the telencephalon and diencephalon of the rabbit. II. An autoradiographic and horseradish peroxidase study of the efferent connections of the anterior olfactory nucleus in the rabbit. J Comp Neurol 164:389-410.

Broadwell RD, Jacobowitz DM (1976) Olfactory relationships of the telencephalon and diencephalon in the rabbit. III. The ipsilateral centrifugal fibers to the olfactory bulbar and retrobulbar formations. J Comp Neurol 170:321-346.

Cole AE, Nicoll RA (1984) The pharmacology of cholinergic excitatory responses in hippocampal pyramidal cells. Brain Res 305:283-290.

Davis BJ, Macrides F (1981) The organization of centrifugal projections from the anterior olfactory nucleus, ventral hippocampal rudiment, and piriform cortex to the main olfactory bulb in the hamster: an autoradiographic study. J Comp Neurol 203:475-493.

Divac I (1975) Magnocellular nuclei of the basal forebrain project to neocortex, brain stem, and olfactory bulb. Review of some functional correlates. Brain Res 93:385-398.

Dolphin AC, Scott RH (1987) Calcium channel currents and their inhibition by $(-)$ baclofen in rat sensory neurones: modulation by guanine nucleotides. J Physiol (Lond) 386:1-17.

Eccles JC, Eccles RM, Magni F (1961) Central inhibitory action attributable to presynaptic depolarization produced by muscle afferent volleys. J Physiol (Lond) 159:147-166.

Eccles JC, Magni F, Willis WD (1962a) Depolarization of central terminals of Group I afferent fibers from muscle. J Physiol (Lond) 160:62-93.

Eccles JC, Schmidt RF, Willis WD (1962b) Presynaptic inhibition of the spinal monosynaptic reflex pathway. J Physiol (Lond) 161:282297.

Eccles JC, Schmidt RF, Willis WD (1963) Pharmacological studies on presynaptic inhibition. J Physiol (Lond) 168:500-530.

Hounsgaard J (1978) Presynaptic inhibitory action of acetylcholine in area CAl of the hippocampus. Exp Neurol 62:787-797.

Krnjevic K (1981) Acetylcholine as modulator of amino-acid-mediated synaptic transmission. In: The role of peptides and amino acids as neurotransmitters, pp 127-141. New York: Liss.

Krnjevic K, Ropert N (1982) Electrophysiological and pharmacological characteristics of facilitation of hippocampal population spikes by stimulation of the medial septum. Neuroscience 7:2165-2183.

Krnjevic K, Reiffenstein RJ, Ropert N (1981) Disinhibitory action of acetylcholine in the rat's hippocampus: extracellular observations. Neuroscience 6:2465-2474.

Kucharski D, Hall WG (1987) New routes to early memories. Science 238:786-788.

Kucharski D, Johanson IB, Hall WG (1986) Unilateral olfactory conditioning in 6 day old rat pups. Behav Neural Biol 46:472-490.

Luskin MB, Price JL (1983) The topographic organization of association fibers of the olfactory system in the rat, including centrifugal fibers to the olfactory bulb. J Comp Neurol 216:264-291.

Macrides F, Davis BJ (1983) The olfactory bulb. In: Chemical neuroanatomy (Emson PC, ed), pp 391-426. New York: Raven.

Macrides F, Davis BJ, Youngs WM, Nadi NS, Margolis FL (1981) Cholinergic and catecholaminergic afferents to the olfactory bulb in the hamster: a neuroanatomical, biochemical and histochemical investigation. J Comp Neurol 203:495-514.

Macrides F, Eichenbaum HB, Forbes WB (1982) Temporal relationship between sniffing and the limbic rhythm during odor discrimination reversal learning. J Neurosci 2:1705-1717.

McLennan H (1971) The pharmacology of inhibition of mitral cells in the olfactory bulb. Brain Res 29:177-184.

Melander T, Staines WA, Hokfelt T, Rokaeus A, Eckenstein F, Salvaterra PM, Wainer BH (1985) Galanin-like immunoreactivity in cholinergic neurons of the septum-basal forebrain complex projecting to the hippocampus. Brain Res 360:130-138.

Mesulam M-M, Mufson EJ, Waincr BH, Levey AI (1983) Central cholinergic pathways in the rat: an overview based on an alternate nomenclature (Ch1-Ch6) Neuroscience 10:1185-1201.

Mori K, Takagi SF (1978) Activation and inhibition of olfactory bulb neurons by anterior commissure volleys in the rabbit. J Physiol (Lond) 729:589-604.

Moyano HF, Molina JC (1982) Olfactory connections of the substantia innominata and nucleus of the horizontal limb of the diagonal band in the rat: an electrophysiological study. Neurosci Lett 34:241-246.

Nakashima M, Mori K, Takagi SF (1978) Centrifugal influence on olfactory bulb activity in the rabbit. Brain Res 154:301-316.

Nickell WT, Shipley MT (1988) Neurophysiology of magnocellular forebrain inputs to the olfactory bulb in the rat: frequency potentiation 
of field potentials and inhibition of output neurons. J Neurosci 8:44924502.

Nickell WT, Shipley MT (1992) Neurophysiology of the olfactory bulb. In: Science of olfaction (Serby MJ, Chobor KL, eds), pp 172-212. New York: Springer.

Paxinos G, Watson C (1986) The rat brain in stereotaxic coordinates, 2d ed. New York: Academic.

Phillips CG, Powell TPS, Shepherd GM (1963) Responses of mitral cells to stimulation of the lateral olfactory tract in the rabbit. J Physio (Lond) 168:65-88.

Rall W, Shepherd GM (1968) Theoretical reconstruction of field potentials and dendrodendritic synaptic interactions in olfactory bulb. J Neurophysiol 31:884-915.

Rovira C, Ben-Ari Y, Cherubini E, Krnjevic K, Ropert N (1983) Pharmacology of the dendritic action of acetylcholine and further observations on the somatic disinhibition in the rat hippocampus in situ. Neuroscience 8:97-106.

Rye DB, Wainer BH, Mesulam M-M, Mufson EJ, Saper CB (1984) Cortical projections arising from the basal forebrain: a study of cholinergic and non-cholinergic components employing combined retrograde tracing and immunohistochemical localization of cholineacetyltransferase. Neuroscience 13:627-643.

Schoenfeld TA, Macrides F (1984) Topographic organization of connections between the main olfactory bulb and pars externa of the anterior olfactory nucleus in the hamster. J Comp Neurol 227:121135.

Shipley MT, Reyes P (1991) Anatomy of the olfactory bulb and central olfactory pathways. In: The human olfactory system (Laing DG, Doty RI, Breipohl W, eds), pp 29-60. Berlin: Springer.

Shute CCD, Lewis PR (1967) The ascending cholinergic reticular system: neocortical, olfactory, and subcortical projections. Brain 90:497520 .
Sofroniew MV, Pearson RCA, Powell TPS (1987) The cholinergic nuclei of the basal forebrain of the rat: normal structure, development, and experimentally induced degeneration. Brain Res 411:310-331.

Takeuchi A, Takeuchi N (1966) On the permeability of the presynaptic terminal of the crayfish neuromuscular junction during synaptic inhibition and the action of $\gamma$-aminobutyric acid. J Physiol (Lond) 183: 433-449.

Teitelbaum H (1971) Lateralization of early memory in the split brain rat. J Comp Physiol 76:51-56.

Valentino RJ, Dingledine R (1981) Presynaptic inhibitory effect of acetylcholine in the hippocampus. J Neurosci 1:784-792.

Van Ooteghem SA, Shipley MT (1984) Factors affecting the sensitivity and consistency of the Koelle-Friedenwald histochemical method for localization of acetylcholinesterase. Brain Res Bull 12:543-553.

Wainer BH, Levey AI, Rye DB, Mesulam MM, Mufson BJ (1985) Cholinergic and non-cholinergic septohippocampal pathways. Neurosci Lett 54:45-92.

Wall PD (1958) Excitability changes in afferent fibre terminations and their relation to slow potentials. J Physiol (Lond) 142:1-21.

Walsh RR (1959) Olfactory bulb potentials evoked by electrical stimulation of the contralateral bulb. Am J Physiol 196:327-329.

Yamamoto C, Kawai N (1967) Presynaptic action of acetylcholine in thin sections from the guinea pig dentate gyrus in vitro. Exp Neurol 19:176-187.

Yamamoto C, Yamamoto T, Wama KI (1963) The inhibitory systems in the olfactory bulb studied by intracellular recording. J Neurophysiol 26:403-415.

Zaborszky L, Carlsen J, Brashear HR, Heimer L (1986) Cholinergic and GABAergic afferents to the olfactory bulb in the rat with special emphasis on the projection from the horizontal limb of the diagonal band. J Comp Neurol 243:488-509. 